\title{
Sobrevida em 10 anos em mulheres com câncer de mama: coorte história de 2000-2014
}

\author{
Survival rate of 10 years among women with breast cancer: \\ a historic cohort from 2000-2014
}

Arlene Laurenti Monterrosa Ayala (https://orcid.org/0000-0002-0466-6374) ${ }^{1}$

Juliana Cristine dos Anjos (https://orcid.org/0000-0002-0919-8389) ${ }^{2}$

Geraldo Antonio Cassol (https://orcid.org/0000-0001-6203-4183) ${ }^{3}$

Doroteia Aparecida Höfelmann (https://orcid.org/0000-0003-1046-3319) ${ }^{4}$

${ }^{1}$ Instituto Luterano de Joinville. Rua Princesa Isabel 438, Centro. 89201-270 Joinville SC Brasil. alayala@bol.com.br ${ }^{2}$ Universidade Federal de Santa Catarina. Florianópolis SC Brasil.

${ }^{3}$ Prefeitura Municipal de Joinville. Joinville SC Brasil.

${ }^{4}$ Departamento de Nutrição, Universidade Federal do Paraná. Curitiba PR Brasil.

\begin{abstract}
Breast cancer is a public health problem due to its high incidence, morbidity and mortality. The analysis of survival for this disease contributes to the description of behavior and prognostic factors. The scope of this article is to investigate survival for 10 years after diagnosis and the prognostic factors of women with breast cancer admitted to the Unified Health System Mastology Service in Joinville, State of Santa Catarina, between 2000 and 2014. A historical cohort study with data from the medical records and death certificates of 1,321 women, of whom 471 were considered eligible under the minimum follow-up criteria of 10 years $(n=288)$, and/or death before this period $(n=183)$. Survival analysis using the Kaplan-Meier model, the Log-Rank test and the Cox regression model was conducted. Overall survival at 10 years was $41 \%$ (CI 95\%, 36.1\%$45.0 \%)$. The risk of 10-year mortality stratified by tumor staging was higher among women with lymphatic invasion and staging II, and staging III, at 60 years or older. The findings suggest that the presence of lymphatic invasion, advanced age and intermediate/advanced staging of the disease can be considered indicators of a worse prognosis for breast cancer.
\end{abstract}

Key words Breast neoplasms, Neoplasm staging, Survival analysis
Resumo O câncer de mama é um problema de saúde pública por sua elevada incidência, morbidade e mortalidade. A análise de sobrevida dessa doença contribui para a descrição do comportamento e dos fatores prognósticos. O objetivo deste artigo é investigar a sobrevida em 10 anos após o diagnóstico, e os fatores prognósticos de mulheres com câncer de mama admitidas no Serviço de Mastologia do Sistema Único de Saúde em Joinville, Santa Catarina, entre 2000-2014. Estudo de coorte histórica com dados dos prontuários e das declarações de óbito de 1.321 mulheres, sendo que 471 foram consideradas elegiveis nos critérios tempo de acompanhamento mínimo de 10 anos $(n=288)$, elou óbito antes deste período $(n=$ 183). Análise de sobrevida realizada pelo modelo de Kaplan-Meier, Teste de Log-Rank e Regressão de Cox. A sobrevida global em 10 anos foi 41,0\% (IC95\% 36,1\% 45,0\%). O risco de mortalidade em 10 anos estratificado pelo estadiamento tumoral foi maior entre as mulheres com invasão linfática e estadiamento II, e com 60 anos ou mais e estadiamento III. Os achados sugerem que a presença de invasão linfática,idade avançada $e$ estadiamento intermediário/avançado da doença podem ser considerados indicadores de pior prognóstico para o câncer de mama.

Palavras-chave Neoplasias da mama, Estadiamento de neoplasias, Análise de sobrevida 


\section{Introdução}

O câncer de mama é considerado um problema de saúde pública devido à sua elevada incidência, morbidade e mortalidade. No ano de 2012 foram estimados 522 mil óbitos por este tipo de câncer em todo mundo, sendo a principal causa de morte nas regiões menos favorecidas, com 324 mil óbitos e ocupando o segundo lugar nas regiões mais desenvolvidas economicamente, com 198 mil, atrás somente do câncer de pulmão ${ }^{1}$.

No Brasil foram registrados 14 óbitos por câncer de mama a cada 100 mil mulheres no ano de 2013 e são esperados aproximadamente 58 mil novos casos de câncer de mama para o biênio 2016-2017, correspondendo a $28,1 \%$ das ocorrências de câncer na população. O risco estimado é de 56,2 casos a cada 100 mil mulheres, sendo o tipo de câncer mais frequente - sem considerar os tumores de pele não melanoma - nas mulheres das Regiões Sul (74,30/100 mil), Sudeste $(68,08 / 100$ mil), Centro-Oeste (55,87/100 mil) e Nordeste (38,74/100 mil). Já na Região Norte, o câncer de mama é o segundo mais incidente, com 22,26 casos por 100 mil mulheres ${ }^{2}$.

A análise de sobrevida do câncer de mama em base populacional contribui para a descrição do comportamento da doença e dos fatores prognósticos a ela relacionados. Dentre os fatores que mais se destacam no prognóstico das mulheres com câncer de mama estão o estadiamento da doença ${ }^{3-5}$, o tamanho do tumor ${ }^{6-8}$, a idade ao diagnóstico ${ }^{9}$, as abordagens terapêuticas utilizadas $^{10,11}$, o atraso no início do tratamento ${ }^{12,13}$, a posse de plano de saúde privado ${ }^{14,15}$, a raça ${ }^{4} \mathrm{e}$ a posição socioeconômica ${ }^{16}$. Estudos apontam que em mulheres diagnosticadas com câncer de mama no estadiamento I/II, a taxa de sobrevida em 5 anos varia entre $80 \%$ e $99 \%$; enquanto em mulheres em estádios mais avançados, que desenvolveram metástase à distância, a taxa de sobrevida em 5 anos cai para menos de $30 \%{ }^{17,18}$.

No Brasil, a sobrevida em 5 anos aumentou de $78 \%$ entre 1995 e 1999 para $87 \%$ entre 2005 e $2009^{19}$. Por outro lado, poucos estudos investigaram a sobrevida em períodos maiores de tempo, o período usual é até cinco anos ${ }^{3,7,15,17}$. Uma análise de sobrevida realizada no período de 10 anos entre mulheres com câncer de mama de Joinville (SC) verificou sobrevida de $83,1 \%$ (IC 95\% 74,1 a $89,3 \%$ ). Dentre os fatores investigados após análise ajustada, apenas o estadiamento avançado esteve significativamente associado à piora da sobrevida em 10 anos $^{5}$. Semelhantemente, a pesquisa desenvolvida com 252 mulheres residentes no Rio Grande do Sul constatou sobrevida em 10 anos de $78,7 \%$, sendo que as probabilidades de sobrevida reduziram conforme o estadiamento da doença, chegando a $0 \%$ quando diagnosticada no estádio IV ${ }^{20}$.

As invasões linfática e/ou sanguínea são os fatores mais relacionados com a metástase linfonodal e, no caso do câncer de mama, estas têm importante papel na previsão do comprometimento metastático axilar, constituindo um importante fator prognóstico ${ }^{21}$. Outro potencial fator prognóstico é o grau histológico. A pesquisa que avaliou mulheres com carcinoma invasivo de mama submetidas à cirurgia no Instituto Nacional de Câncer identificou menor sobrevida para as pacientes com tumores pouco diferenciados em relação àquelas com tumores bem ou moderadamente diferenciados ${ }^{6}$. Entretanto, há controvérsias, o estudo de Moraes et al. ${ }^{20}$, de base hospitalar, ao investigar 286 mulheres verificou que o grau histológico não mostrou significância na avaliação da curva de sobrevida para os diferentes graus de diferenciação analisados.

É certo que há avanços no diagnóstico do câncer de mama, o que permite identificar a doença em estádios mais precoces, contudo, muitas mulheres ainda descobrem a doença em estadiamento avançado, o que limita o sucesso terapêutico e aumenta o risco de mortalidade ${ }^{17}$. $\mathrm{O}$ número de sobreviventes de câncer tem aumentado, e também o interesse em reconhecer os fatores associados à maior sobrevida e qualidade de vida das pacientes ${ }^{22}$. Diante do exposto e considerando a relevância do tema, este estudo tem por objetivo investigar por meio de uma coorte histórica, a sobrevida em 10 anos e seus fatores prognósticos de mulheres com câncer de mama admitidas no Serviço de Mastologia do Sistema Único de Saúde (SUS) em Joinville, Santa Catarina, entre 2000-2014.

\section{Métodos}

Estudo de coorte histórica com mulheres de uma unidade de saúde - Policlínica do Boa Vista - do Sistema Único de Saúde, considerada centro de referência para tratamento do câncer de mama e localizada em Joinville, Santa Catarina.

Joinville está situada na região nordeste do Estado de Santa Catarina, com população estimada em 2016 de 569.645 habitantes. Trata-se do município mais populoso de Santa Catarina, terceiro da região Sul e o $3^{\circ}$ maior polo industrial da Região Sul do Brasil ${ }^{23}$. A cidade possui um dos 
mais altos índices de desenvolvimento humano (IDH) entre os municípios brasileiros $(0,809)$, ocupando a vigésima primeira posição nacional e a quarta entre os municípios catarinenses ${ }^{24}$.

Os dados da presente pesquisa foram coletados dos prontuários e das declarações de óbito do Sistema de Informações sobre Mortalidade (SIM) da Secretaria Municipal de Saúde de Joinville, e inseridos em uma planilha no Excel, desenhada para a investigação.

Como critérios de exclusão, todas as mulheres com estadiamento patológico classificado como Tis (carcinoma in situ) $(n=69)$ ou ignorado $(n=$ 13), bem como, os casos classificados como neoplasias hematológicas $(\mathrm{n}=3)$ ou indeterminadas $(\mathrm{n}=4)$ foram excluídas da amostra.

As variáveis em estudo foram extraídas dos prontuários e das declarações de óbito: faixa etária ao diagnóstico - calculada a partir da data de nascimento e diagnóstico disponíveis no prontuário - (até 59 anos; 60 ou mais), história familiar de câncer de mama (não; sim), data do diagnóstico, estadiamento TNM (Tumor, Linfonodos, Metástases) patológico (I; II; III; IV), grau de Nottingham [bem diferenciado (I); moderadamente diferenciado (II); indiferenciado (III)], invasão linfática (não; sim), invasão sanguínea (não; sim) e óbito ( tadiamento e a classificação TNM foram obtidas a partir do laudo anátomo-patológico proveniente do Centro de Diagnóstico Anátomo Patológico do município.

$\mathrm{O}$ estadiamento das mulheres baseou-se na classificação TNM proposta em 1988 pela Union for International Cancer Control ${ }^{25}$. A classificação considera o tamanho do tumor, o comprometimento dos linfonodos e a presença ou não de metástase no diagnóstico da doença.

As informações sobre os óbitos das mulheres foram obtidas trimestralmente no período de janeiro de 2001 a abril de 2015 a partir dos dados enviados pela Vigilância Epidemiológica do município, e da análise dos prontuários disponíveis no serviço. Foram considerados os óbitos em decorrência do câncer de mama (causa básica) e em consequência do seu tratamento. Mulheres que foram a óbito por causas não relacionadas ao câncer de mama ou ao seu tratamento foram censuradas na data de óbito.

A sobrevida em 10 anos foi calculada considerando o intervalo entre a data do diagnóstico disponível no prontuário e a data do óbito ou final do acompanhamento. Todas as mulheres que permaneceram vivas até a data de 31 de dezembro de 2014 foram censuradas ao final do estudo, bem como aquelas que morreram após 10 anos de acompanhamento. As mulheres que não completaram 10 anos de acompanhamento, sem ter ido a óbito $(\mathrm{n}=779)$, foram excluídas da análise (Figura 1). A Figura 1 apresenta o fluxograma da amostra estudada, considerando os critérios de inclusão e exclusão adotados.

A análise descritiva dos dados foi realizada por meio do cálculo das frequências absolutas (n) e relativas (\%). A sobrevida em 10 anos foi estratificada segundo as categorias das variáveis exploratórias, por meio do modelo de KaplanMeier $^{25}$. Com o teste log-rank foram investigadas as funções de sobrevida, também de acordo com as variáveis exploratórias ${ }^{26}$. E por meio da Regressão de Cox foram estimadas as razões de risco (Hazard Ratios) brutas e ajustadas e os respectivos intervalos de confiança de 95\% (IC95\%). As curvas de sobrevida geradas foram inspecionadas visualmente, e os modelos testados formalmente por meio do teste de proporcionalidade de riscos. Como a hipótese de distribuição proporcional dos riscos de mortalidade foi rejeitada para a variável estadiamento inicial ( $\mathrm{p}<0,05)$, as análises de regressão foram estratificadas para cada estadiamento tumoral. A entrada das variáveis no modelo ajustado iniciou-se pelas variáveis: faixa etária e história familiar de câncer de mama. No segundo modelo foram acrescidas as variáveis: 1) grau de Nottingham; 2) invasão sanguínea e, 3) invasão linfática. $\mathrm{Na}$ análise multivariável considerou-se o valor de $\mathrm{p}<0,25$ para a inclusão das variáveis, as quais foram consideradas estatisticamente significativas quando valor de $\mathrm{p}<0,05$. $\mathrm{O}$ teste de proporcionalidade de riscos foi aplicado após cada modelo. Todas as análises foram realizadas no programa Stata versão 12.

Este estudo foi submetido ao Comitê de Ética em Pesquisa (CEP) da Plataforma Brasil por meio de sistema eletrônico e aprovado pelo CEP do Instituto Superior e Centro Educacional Luterano Bom Jesus/Ielusc, e Certificado de Apresentação para Apreciação Ética (CAAE). A Resolução 466/12 do Conselho Nacional de Saúde (CNS), que trata das Diretrizes e Normas Regulamentadoras de Pesquisas envolvendo Seres Humanos norteou as etapas da pesquisa. Desta forma, foi garantido o sigilo das informações coletadas.

\section{Resultados}

Após adotar os critérios de exclusão restaram 471 mulheres, sendo que 288 completaram 10 anos de acompanhamento, e 183 foram inseridas sem 


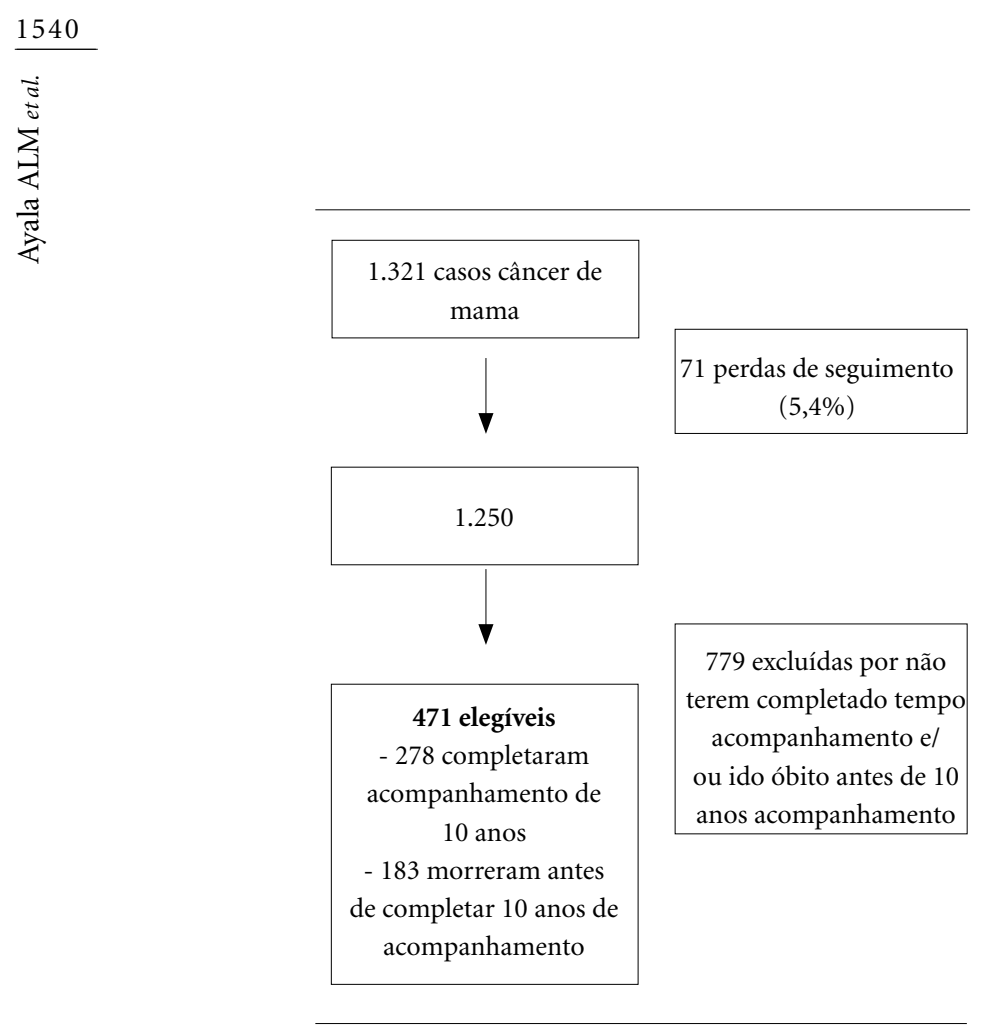

Figura 1. Fluxograma dos casos de câncer de mama de centro de referência para tratamento em Joinville (SC), 2001-2014.

ter concluído o tempo, mas tendo ido a óbito entre os anos de 2000-2014. O tempo médio de acompanhamento para as mulheres que permaneceram vivas e foram acompanhadas pelo tempo mínimo de 120 meses foi de 142,8 meses (DP: 16,2 ), e para aquelas que foram a óbito, o tempo médio de acompanhamento foi de 36,0 meses (DP: 29,8).

A idade média das mulheres ao diagnóstico foi de 55,3 anos (DP: 14,0) e variou de 24,3 a 96,1 anos. A maioria não referiu história familiar de câncer de mama $(85,2 \%)$ e foi classificada no momento do diagnóstico com estadiamento patológico II ou III $(79,1 \%)$. O grau de Nottingham 2 (moderadamente diferenciado) foi observado em 54,5\% das mulheres, 54,8\% foram classificadas com invasão linfática e 70,6\% foram classificadas sem invasão sanguínea (Tabela 1). As curvas de sobrevida em 10 anos, segundo as categorias de cada variável analisada podem ser observadas na Figura 2.

A sobrevida global em 10 anos foi de 41,0\% (IC95\% 36,1\%-45,0\%). A chance de sobrevida foi menor para as mulheres nos estadiamentos III e IV, com grau de Nottingham 2 e 3, e que apresentaram invasão linfática e/ou sanguínea (Tabela 1).

Ao estratificar o risco de mortalidade em 10 anos de acordo com os estadiamentos tumorais iniciais, observou-se que nenhuma das variáveis investigadas esteve associada para o estadiamento I ou IV. Para o estadiamento II, observou-se maior mortalidade entre as mulheres com grau de Nottingham 2 e/ou 3 e com invasão linfática; enquanto no estadiamento III, somente as mulheres com 60 anos ou mais apresentaram maior risco de mortalidade. (Tabela 2 ).

Após análise ajustada, observou-se que o risco de mortalidade em 10 anos permaneceu associado à invasão linfática no estadiamento II e a faixa etária (60 anos ou mais) para o estadiamento. Já a variável invasão sanguínea apresentou-se como fator de proteção para o estadiamento II. Os valores de $\mathrm{p}$ para teste de proporcionalidade de riscos foi superior a 0,05 em todos os modelos ajustados (Tabela 2).

\section{Discussão}

Os resultados do presente estudo indicaram que a taxa de sobrevida global após 10 anos foi de $41,0 \%$. Esta taxa de sobrevida é semelhante àquela observada em um estudo realizado com 277 mulheres com câncer invasivo de mama na cidade de Goiânia, onde a sobrevida global foi de $41,5 \%{ }^{27}$. Entretanto, as taxas de sobrevida global em 10 anos de pacientes com câncer de mama verificada no centro de referência oncológica no município de Juiz de Fora, no estado de Minas Gerais, foi de $56,3 \%{ }^{28}$. Assim como Balabram et al. ${ }^{26}$, ao avaliarem a sobrevida de 897 pacientes com câncer de mama de um hospital público em Belo Horizonte que trata principalmente pacientes do Sistema Único de Saúde, constataram a sobrevida global de $64,5 \%$. Os prováveis fatores associados ao incremento na sobrevida observada nestes dois estudos, em relação à taxa verificada em Joinville, podem decorrer do maior percentual de mulheres classificadas ao diagnóstico nos estadiamentos I e II tanto em Juiz de Fora ${ }^{28}$ quanto em Belo Horizonte ${ }^{26} \mathrm{e}$ devido à maior presença de tumores bem diferenciados em Belo Horizonte $^{26}$, que é uma característica do tumor com melhor prognóstico ${ }^{29}$.

Duas outras pesquisas ${ }^{5,30}$ anteriormente realizadas no mesmo serviço de saúde da cidade de Joinville - do qual é proveniente a coorte analisada neste estudo - encontraram taxas de sobrevida diferentes. Ayala ${ }^{30}$ ao investigar 655 mulheres com câncer de mama no período de 2000 a 2009 constatou sobrevida em 10 anos de 57,8\%. Enquanto Höfelmann et al. ${ }^{5}$ verificaram taxa de sobrevida em 10 anos de 83,1\%, ao analisarem 170 mulheres com câncer de mama entre 2001 a 
Tabela 1. Distribuição das variáveis, óbitos e sobrevida em 10 anos em mulheres com câncer de mama. Joinville, Santa Catarina, Brasil, 2000-2014.

\begin{tabular}{|c|c|c|c|c|}
\hline $\begin{array}{l}\text { Variáveis } \\
\text { Categorias }\end{array}$ & $\begin{array}{c}n(\%) \\
10 \text { anos }\end{array}$ & $\begin{array}{c}\text { Óbitos } \\
10 \text { anos } \\
\text { n }(\%)\end{array}$ & $\begin{array}{c}\text { Sobrevida } \\
10 \text { anos } \\
\% \text { (IC95\%) }\end{array}$ & $\begin{array}{c}\text { Teste } \\
\text { log-rank } \\
\text { (valor de p) }\end{array}$ \\
\hline Faixa etária diagnóstico $(\mathrm{N}=471)$ & & & & 0,012 \\
\hline Até 59 anos & $313(66,5)$ & $178(56,9)$ & $43,1(37,6-48,5)$ & \\
\hline 60 anos ou mais & $158(33,5)$ & $102(64,6)$ & $35,4(28,1-42,9)$ & \\
\hline Câncer na família $(\mathrm{N}=379)$ & & & & 0,808 \\
\hline Não & $323(85,2)$ & $190(58,8)$ & $41,2(35,8-46,5)$ & \\
\hline Sim & $56(14,8)$ & $34(60,7)$ & $39,3(26,6-51,7)$ & \\
\hline Estadiamento patológico $(\mathrm{N}=454)$ & & & & $<0,001$ \\
\hline I & $61(13,4)$ & $11(18,0)$ & $82,0(69,8-89,6)$ & \\
\hline II & $185(40,8)$ & $73(39,5)$ & $60,5(53,1-67,2)$ & \\
\hline III & $174(38,3)$ & $155(89,1)$ & $10,9(6,8-16,1)$ & \\
\hline IV & $34(7,5)$ & $34(100,0)$ & - & \\
\hline Grau de Nottingham $(\mathrm{N}=363)$ & & & & 0,002 \\
\hline 1 & $33(9,1)$ & $13(39,4)$ & $60,6(42,0-74,5)$ & \\
\hline 2 & $198(54,5)$ & $109(55,0)$ & $44,9(37,9-51,7)$ & \\
\hline 3 & $132(36,4)$ & $92(69,7)$ & $30,3(22,7-38,2)$ & \\
\hline Invasão linfática $(\mathrm{N}=374)$ & & & & $<0,001$ \\
\hline Não & $169(45,2)$ & $65(38,5)$ & $61,5(53,8-68,4)$ & \\
\hline Sim & $205(54,8)$ & $150(73,2)$ & $26,8(21,0-33,0)$ & \\
\hline Invasão sanguínea $(\mathrm{N}=374)$ & & & & 0,002 \\
\hline Não & $264(70,6)$ & $141(53,4)$ & $46,6(40,5-52,5)$ & \\
\hline Sim & $110(29,4)$ & $74(67,3)$ & $32,7(24,2-41,5)$ & \\
\hline
\end{tabular}

2011. Dentre as potenciais explicações para o aumento da sobrevida entre as usuárias do mesmo serviço nos dois recortes temporais empregados e com sobreposição de períodos, está a inclusão de casos prevalentes, de mulheres que passaram a ser acompanhadas pelo serviço anos após o diagnóstico, e que iniciaram o tratamento em outros locais.

Nas últimas décadas, o aumento do acesso aos programas de rastreamento tem levado ao incremento na detecção de casos de câncer de mama em estádios mais iniciais. Contudo, é importante destacar que o aumento da detecção desses casos mais iniciais via programas de rastreamento, não necessariamente está ligado à redução da mortalidade pela doença. Ao invés disto, há evidências de que ele pode aumentar a incidência de câncer, pois, casos que talvez nunca viessem a apresentar manifestações clínicas da doença passam a ser tratados, e contribuem artificialmente para o aumento da sobrevida ${ }^{31,32}$. Nos Estados Unidos, por exemplo, de 1976 a 2008, Bleyer e Welch ${ }^{32}$ verificaram que após excluir o aumento transitório da incidência de câncer de mama associado ao uso da terapia de reposição hormonal, e após ajustar para as tendências da incidência de câncer em mulheres com menos de 40 anos de idade, tumores que foram detectados em virtude do rastreamento, e que poderiam nunca vir a apresentar manifestações clínicas, foram diagnosticados em $31 \%$ dos casos de câncer de mama no período ${ }^{32}$.

A maioria das mulheres avaliadas neste estudo foi classificada com estadiamento patológico inicial II ou III, com grau do tumor moderadamente diferenciado, com invasão linfática e sem invasão sanguínea. No entanto, as menores taxas de sobrevida foram observadas dentre aquelas nos estádios III ou IV, com grau de tumor pouco diferenciado e apresentando invasão linfática e/ou sanguínea. Vários estudos confirmam estes achados $^{20,33,34}$. Neste estudo, a sobrevida em 10 anos para o estádio II foi de $60,5 \%$, para o estádio III foi de $10,9 \%$ e para o IV foi de $0 \%$. O estudo de Fayer et al. ${ }^{28}$ descreveu uma taxa de sobrevivência em 10 anos de $63 \%$ para o estádio II, 26,3\% para o estádio III e 14,3\% para o estádio IV. Balabram et al. ${ }^{26}$, ao investigarem mulheres com câncer de mama operável (estágios I-III), mostraram uma sobrevida em 10 anos de 69,8\% para o estádio II e $43 \%$ para o estádio III. Nos Estados Unidos, no período entre 1985 e 1995 foram verificadas taxas de sobrevida em 10 anos relativas aos estádios III 


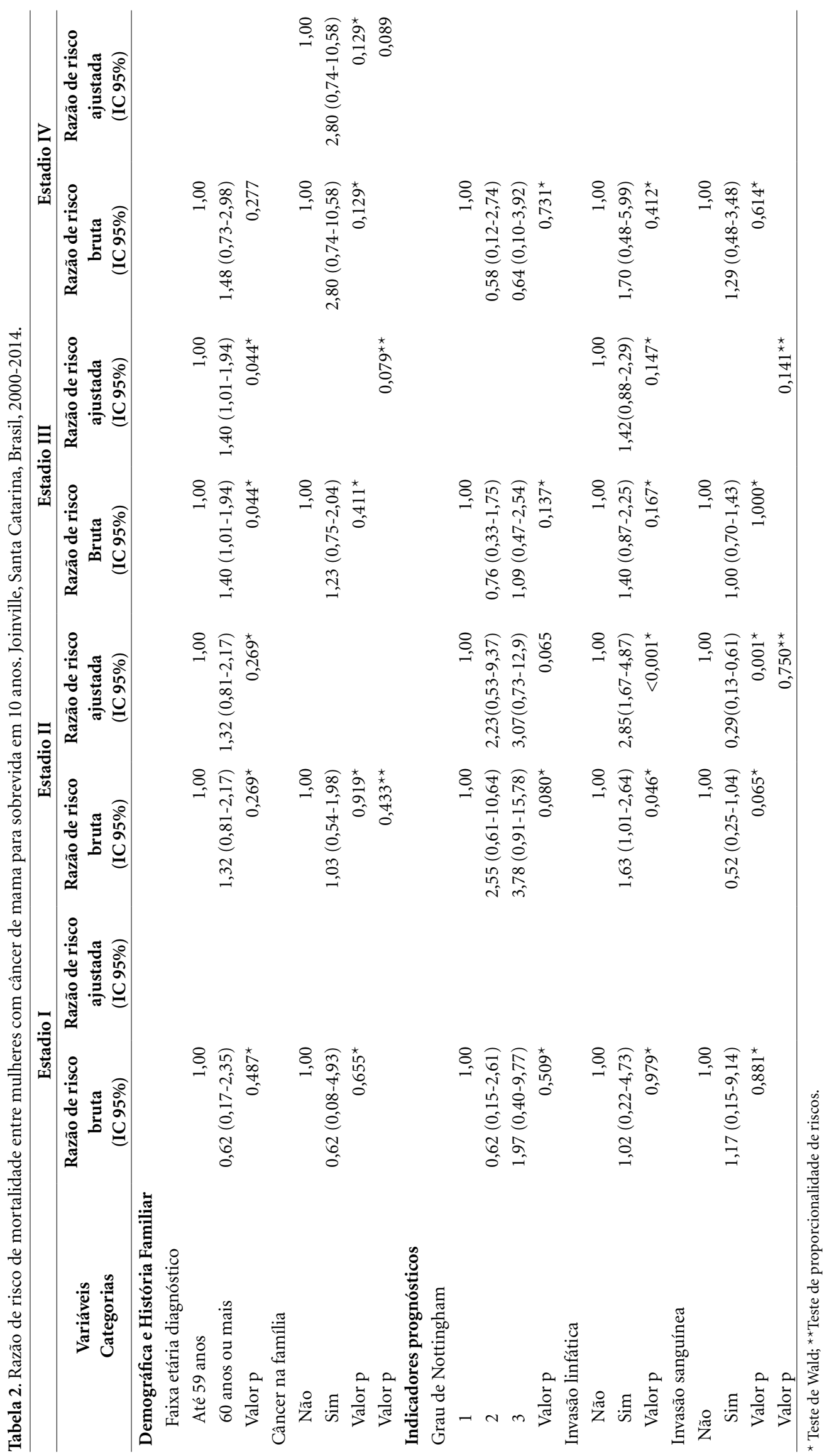


a) Faixa etária

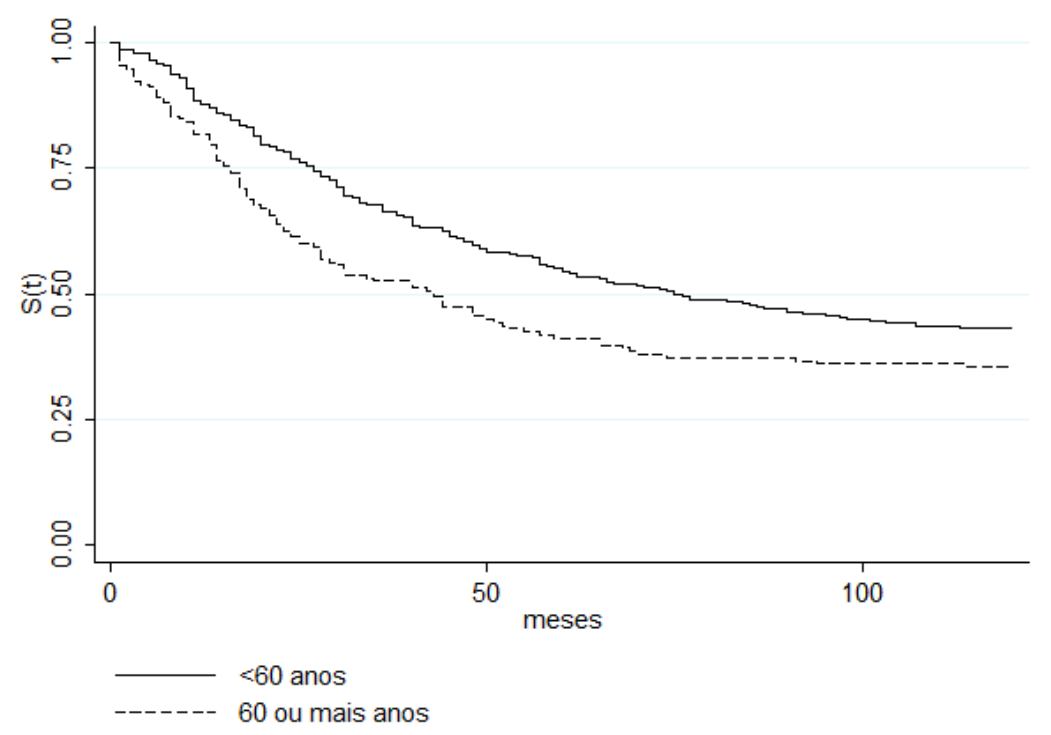

b) História familiar

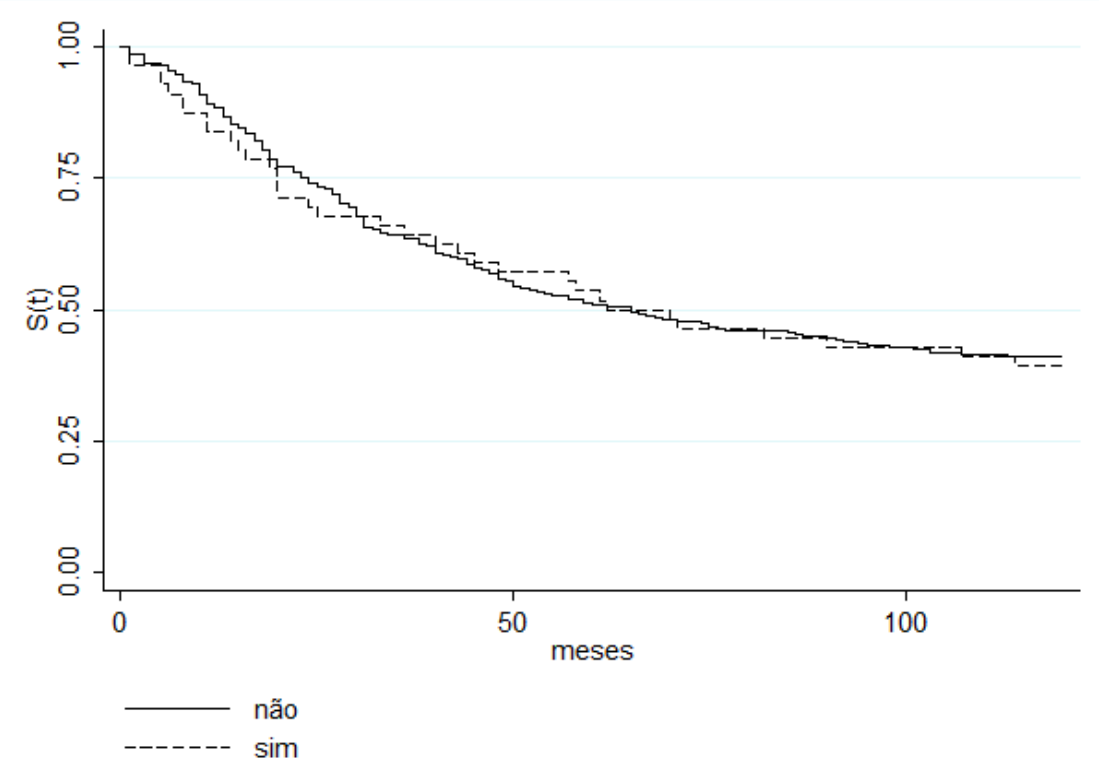

Figura 2. Curvas de sobrevida em 10 anos em mulheres com câncer de mama, segundo variáveis. Joinville, Santa Catarina, Brasil, 2000-2014.

e IV, de $36 \%$ e $7 \%$, respectivamente ${ }^{34}$. Moraes et al. ${ }^{20}$ observaram probabilidade de sobrevida em 10 anos em conformidade à encontrada neste estudo, que foi de $0 \%$ entre brasileiras diagnosticadas com câncer de mama no estádio inicial IV. As diferenças na sobrevida em cada um desses estudos podem ser em virtude das diferentes me- todologias e composição de amostras adotadas, bem como das características tumorais e do tratamento utilizado.

O estadiamento do tumor afeta diretamente a taxa de sobrevida do portador de câncer de mama, visto que as características e o tamanho do tumor, assim como a condição e o compro- 
c) Estadiamento inicial

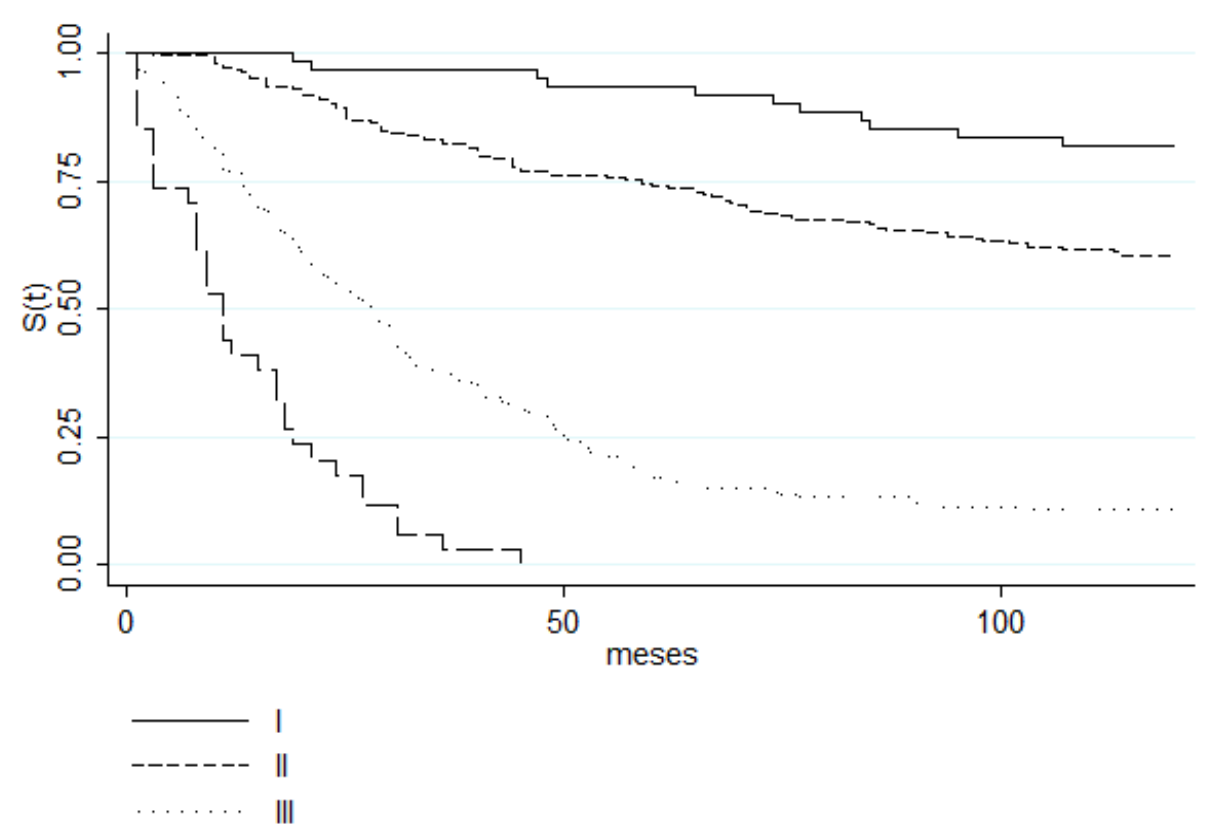

d) Grau de Nottingham

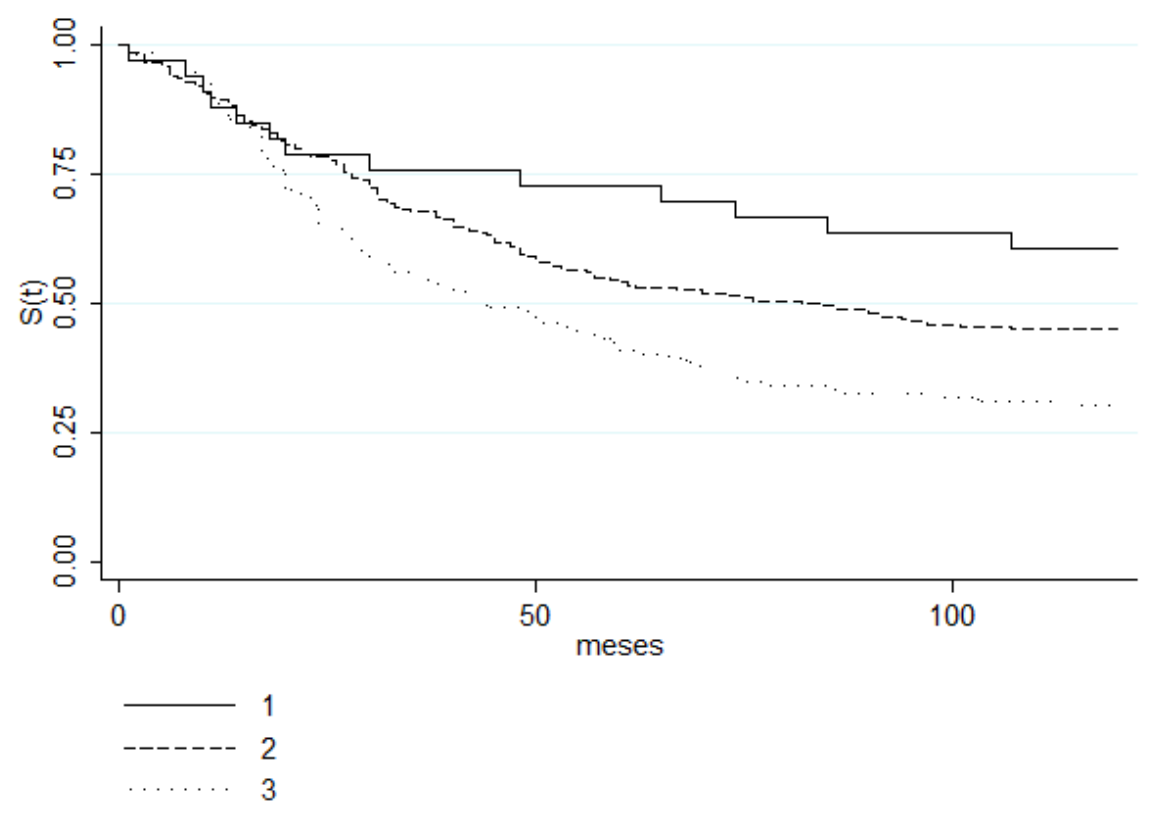

Figura 2. Curvas de sobrevida em 10 anos em mulheres com câncer de mama, segundo variáveis. Joinville, Santa Catarina, Brasil, 2000-2014.

metimento dos linfonodos da região axilar representam importantes indicadores do prognóstico da doença ${ }^{8,35,36}$. O grau histológico compreende a malignidade do tumor, refletindo a sua maior ou menor capacidade de metastatização $0^{37}$. Mu- lheres com grau de Nottingham 3 apresentaram as piores taxas de sobrevida em 10 anos. Estudos que tenham analisado o impacto do grau histológico do câncer de mama na sobrevida de portadores da doença são escassos, suas taxas variam 
e) Invasão linfática

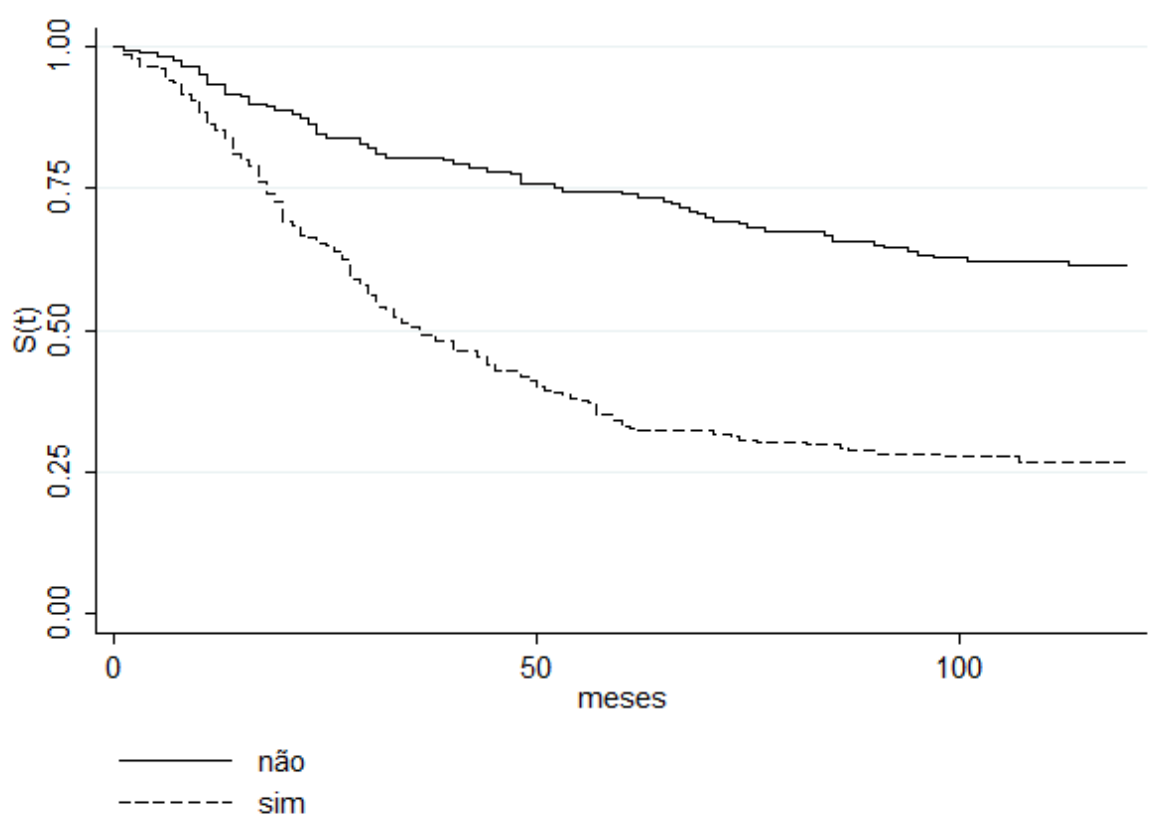

f) Invasão sanguínea

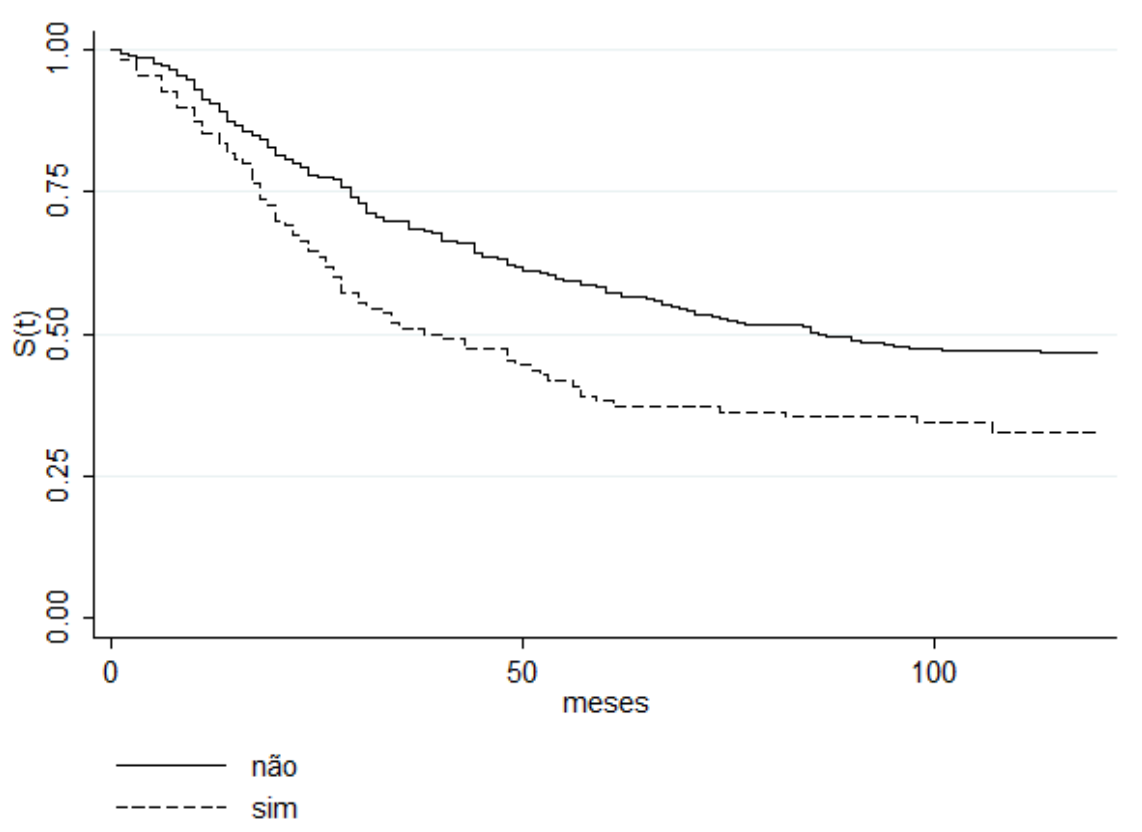

Figura 2. Curvas de sobrevida em 10 anos em mulheres com câncer de mama, segundo variáveis. Joinville, Santa Catarina, Brasil, 2000-2014.

de acordo com o tempo de sobrevida avaliado e os resultados são controversos ${ }^{20,38}$, dificultando a sua determinação. Contudo, uma coorte hospitalar realizada na cidade do Rio de Janeiro entre
1992 e 1996 encontrou associação entre o grau histológico e a sobrevida em 5 anos, com taxas de sobrevida de $94 \%$ entre pacientes com tumores grau 1, 82\% entre aquelas com tumores grau 2 e 
a) Faixa etária

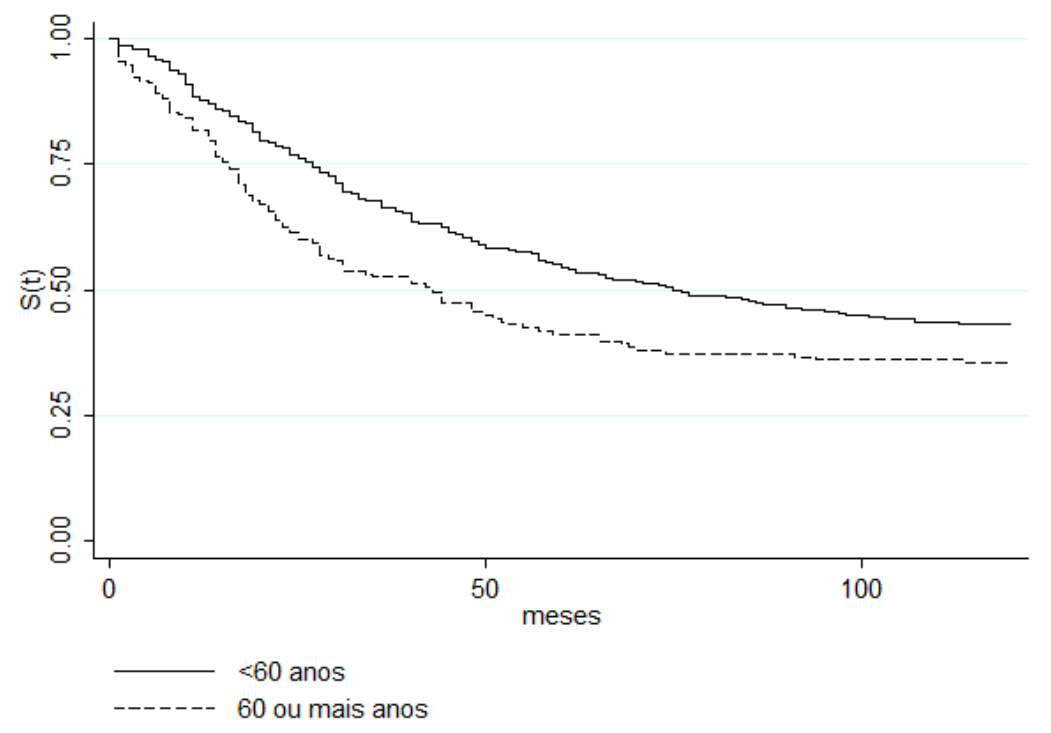

b) História familiar

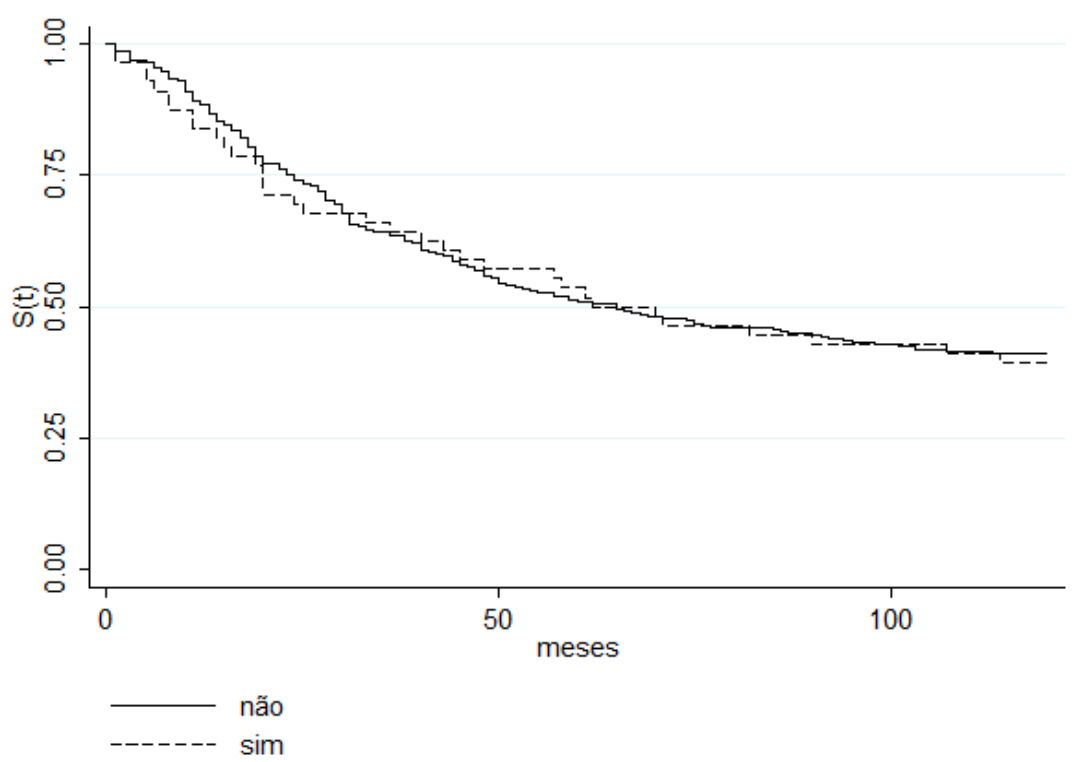

Figura 2. Curvas de sobrevida em 10 anos em mulheres com câncer de mama, segundo variáveis. Joinville, Santa Catarina, Brasil, 2000-2014.

$65 \%$ de sobrevida entre as mulheres com tumores grau $3^{38}$. Já uma pesquisa desenvolvida em Santa Maria, no Rio Grande do Sul, não encontrou significância estatística entre o grau histológico do tumor e a taxa de sobrevida em 5 ou 10 anos $^{20}$.

Quanto às invasões linfática e sanguínea, após a análise ajustada, a primeira apresentou significância estatística de acordo com o estadia- mento II, e a segunda apresentou-se como fator de proteção, também para o estadiamento II da doença, em relação à sobrevida em 10 anos. $\mathrm{O}$ valor prognóstico da invasão linfática e/ou sanguínea foi confirmado em outros estudos ${ }^{39-41}$. Mohammed et al. ${ }^{39}$ verificaram sobrevida global em 20 anos de $72 \%$ para as mulheres sem invasão linfática, em comparação com 54\% em pacien- 
tes com esta invasão. Segundo Hwang et al. ${ }^{41}$, a presença de invasão linfática e sanguínea é fator preditivo para a disseminação neoplásica, levando, consequentemente, ao pior prognóstico dentre os portadores da doença. No entanto, estudos sugerem que esta associação ainda não está bem esclarecida ${ }^{42,43}$.

No que diz respeito à idade, menores taxas de sobrevida foram observadas entre as mulheres idosas, sendo que o risco de morte em 10 anos foi maior para o estadiamento III. Vários estudos têm demonstrado que as mulheres idosas têm tido um pior desfecho ${ }^{44-46}$. De acordo com os autores, mulheres mais velhas, apesar de apresentarem características menos agressivas do tumor, frequentemente, possuem comorbidades associadas ou estádios mais avançados da doença ao diagnóstico. O que poderia explicar, em parte, as menores taxas de sobrevida neste grupo etário ${ }^{44,45}$. Porém, a questão do pior prognóstico entre as mulheres idosas é conflitante com estudos que têm evidenciado que as mulheres jovens têm tido um pior prognóstico. Nixon et al. ${ }^{47}$, ao investigarem o câncer de mama entre as mulheres abaixo de 35 anos e em estadiamento I e II, verificaram maior recidiva e metástase à distância quando comparadas às mulheres mais velhas. Esta diferença, de acordo com os autores, pode ser atribuída, em parte, aos fatores associados a um pior prognóstico comumente presente nesta população, dentre eles, pouca diferenciação do tumor, invasão de vasos linfáticos, necrose e negatividade do receptor de estrogênio. Da mesma forma, outros estudos referem que a idade jovem está associada geralmente a características mais agressivas do tumor, sendo, portanto, um fator independente de prognóstico negativo ${ }^{48,49}$. Todavia, há outro estudo que não mostrou diferenças nas taxas de sobrevivência entre pacientes mais jovens e idosos ${ }^{50}$.

Como limitações do presente estudo ressaltam-se a perda de seguimento de algumas mulheres e importantes falhas no preenchimento dos dados. Com relação às falhas no preenchimento de dados, as variáveis câncer de mama na família, grau de Nottingham, invasão linfática e invasão sanguínea - consideradas de importância epidemiológica pelo fato de que a partir delas poder ser traçada a evolução da doença, a fim de estabelecer um melhor planejamento terapêutico, bem como subsidiar estudos de sobrevida - não foram informadas com a completude pertinente à sua importância. As falhas ou o número de casos ignorados para as variáveis câncer de mama na família foram de aproximadamente $15 \%$, o que pode ter enfraquecido o poder explicativo desta variável na análise de sobrevida. Diferentemente das variáveis invasão linfática, invasão sanguínea e grau de Nottingham, que apesar dos casos ignorados representarem 12,4\%, 12,5\% e 15,0\%, respectivamente, estas permaneceram associadas à pior sobrevida.

As falhas no preenchimento não se aplicam somente a este estudo, outros pesquisadores que investigaram o câncer de mama em diferentes localidades do país, a partir do Sistema de Informação do Programa de Controle do Câncer de Mama (SISMAMA), identificaram problemas de exatidão no preenchimento dos dados ${ }^{51-53}$. Silva et al. ${ }^{51}$ ao investigarem a associação entre variáveis sociodemográficas e o estadiamento avançado das neoplasias de mama no Espírito Santo constataram completude muito ruim de dados para algumas variáveis. Completude ruim também foi observada no estudo de Pinto et al. ${ }^{52}$ ao analisarem os dados dos registros hospitalares de câncer no Brasil. Os autores encontraram incompletude para variáveis como: estadiamento da doença, grau de instrução, estádio da doença ao final do primeiro tratamento, entre outras; e os mesmos sugerem que, as falhas nos registros dos dados ocorrem em razão da falta de capacitação de recursos humanos e da inadequação na utilização e divulgação das informações. Afirmando, assim, a necessidade do engajamento dos profissionais de saúde na geração de informações referentes à assistência ao paciente e ao correto e completo fornecimento de dados ${ }^{52}$.

Outro aspecto a ser considerado diz respeito às informações relativas ao tipo de tratamento que não puderam ser obtidas com qualidade, pois a paciente passa a ser acompanhada por um centro de alta complexidade oncológica, o que dificulta o acesso às informações. Adicionalmente, os autores não dispunham de informações para comparar as perdas de seguimento, das demais participantes do estudo, contudo, as perdas foram relativamente pequenas $(5,4 \%)$.

Além disso, os resultados do presente estudo devem ser interpretados com cautela, uma vez que, apesar de a sobrevida ser considerada um desfecho tradicional na área de oncologia, a existência de métodos de rastreamento disponíveis, como ocorre com o câncer de mama, pode levar a um aumento espúrio da sobrevida relacionado ao viés de tempo de antecipação, de tempo de duração e de sobrediagnóstico ${ }^{31}$. A validade externa dos dados também deve ser considerada com precaução, uma vez que as mulheres avaliadas foram usuárias do Sistema Único de Saúde. Não 
é possível precisar as condições socioeconômicas da amostra, pois as informações disponíveis nos prontuários referentes a estas condições não são padronizadas, e não foram extraídas para o presente estudo. Informações da Agência Nacional de Saúde indicam que, em 2014, 40\% da população de Joinville era usuária de planos de saúde suplementar ${ }^{54}$. Considerando que o Serviço de Mastologia do Pam do Boa Vista é a porta de entrada do SUS no município de Joinville para as mulheres com afecções da mama, pode-se estimar que este serviço atende parcela importante dos casos de mulheres com câncer de mama da cidade, e a totalidade de usuárias do SUS com a enfermidade. Adicionalmente, a realização de busca ativa das mulheres pelo serviço, reforça a validade interna dos achados.
Por fim, ressalta-se a contribuição dessa pesquisa na análise e consolidação dos fatores prognósticos que afetam a sobrevivência de mulheres com câncer de mama. O maior risco de mortalidade entre mulheres idosas e estadiamento intermediário/avançado foi observado, no entanto, sabese que o comportamento da sobrevida em relação à faixa etária ainda é controverso, assim como o impacto das variáveis invasão sanguínea e linfática na sobrevida ainda é inconclusivo e pouco explorado. Desta forma, sugere-se que futuros estudos aprofundem o papel destas variáveis, com o intuito de definir melhor o prognóstico e o tratamento das mulheres portadoras de câncer de mama. Além da necessidade de novas pesquisas, tanto em nível local quanto em nível estadual e nacional, que avaliem, sobretudo, a assistência em saúde prestada no Brasil a este segmento populacional.

\section{Colaboradores}

ALM Ayala, JC Anjos, GA Cassol e DA Hofelmann participaram da concepção e delineamento do estudo, interpretação dos dados, e redação. ALM Ayala, JC Anjos, GA Cassol e DA Hofelmann aprovaram a versão final do manuscrito, e são responsáveis por todos os seus aspectos, incluindo a garantia de sua integridade. 


\section{Referências}

1. World Health Organization (WHO). International Agency for Research on Cancer. GLOBOCAN 2012: Estimated Cancer Incidence, Mortality and Prevalence Worldwide in 2012. [acessado 2016 Jun 15]. Disponível em: http://globocan.iarc.fr/Pages/online.aspx.

2. Instituto Nacional de Câncer José Alencar Gomes da Silva (INCA). Estimativa 2016: incidência de câncer no Brasil. Rio de Janeiro: INCA; 2015. [acessado 2016 Jun 15]. Disponível em: http://www.inca.gov.br/bvscontrolecancer/publicacoes/edicao/Estimativa_2016.pdf.

3. Sant M, Allemani C, Capocaccia R, Hakulinen T, Aareleid T, Coebergh JW, Coleman MP, Grosclaude P, Martinez C, Bell J, Youngson J, Berrino F; EUROCARE Working Group. Stage at diagnosis is a key explanation of differences in breast cancer survival across Europe. Int J Cancer 2003; 106(3):416-422.

4. Siegel R, Naishadham D, Jemal A. Cancerstatistics, 2012. CA Cancer J Clin 2012; 62(1):10-29.

5. Höfelmann DA, Anjos JC, Ayala AL. Sobrevida em dez anos e fatores prognósticos em mulheres com câncer de mama em Joinville, Santa Catarina, Brasil. Cien Saude Colet 2014; 19(6):1813-1824.

6. Mendonça GAS, Silva AM, Caula WM. Características tumorais e sobrevida de cinco anos em pacientes com câncer de mama admitidas no Instituto Nacional de Câncer, Rio de Janeiro, Brasil. Cad Saude Publica 2004; 20(5):1232-1239.

7. Kim KJ, Huh SJ, Yang JH, Park W, Nam SJ, Kim JH, Lee JH, Kang SS, Lee JE, Kang MK, Park YJ, Nam HR. Treatment results and prognostic factors of early breast cancer treated with a breast conserving operation and radiotherapy. Jpn J Clin Oncol 2005; 35(3):126-133.

8. Guerra MR, Mendonça GAS, Bustamante-Teixeira MT, Cintra JRD, Carvalho LM, Magalhães LMPV. Sobrevida de cinco anos e fatores prognósticos em coorte de pacientes com câncer de mama assistidas em Juiz de Fora, Minas Gerais, Brasil. Cad Saude Publica 2009; 25(11):2455-2466.

9. Anderson WF, Jatoi I, Devesa SS. Distinct breast cancer incidence and prognostic patterns in the NCI's SEER program: suggesting a possible link between etiology and outcome. Breast Cancer Res Treat 2005; 90(2):127137.

10. Panades M, Olivotto IA, Speers CH, Shenkier T, Olivotto TA, Weir L, Allan SJ, Truong PT. Evolving treatment strategies for inflammatory breast cancer: a population-based survival analysis. J Clin Oncol 2005; 23(9):1941-1950.

11. Hooning MJ, Aleman BMP, van Rosmalen AJM, Kuenen MA, Klijn JGM, van Leeuwe FE. Cause-specific mortality in long-term survivors of breast cancer: a 25year follow-up study. Int J Radiat Oncol Biol Phys 2006; 64(4):1081-1091.

12. Hébert-Croteau N, Freeman CR, Latreille J, Rivard M, Brisson J. A population-based study of the impact of delaying radiotherapy after conservative surgery for breast cancer. Breast Cancer Res Treat 2004; 88(2):187196.

13. Lohrisch C, Paltiel C, Gelmon K, Speers C, Taylor S, Barnett J, Olivotto IA. Impact on survival of time from definitive surgery to initiation of adjuvant chemotherapy for early-stage breast cancer. J Clin Oncol 2006; 24(30):4888-4894.
14. Ward E, Halpern M, Schrag N, Cokkinides V, De Santis C, Bandi P, Siegel R, Stewart A, Jemal A. Association of insurance with cancer care utilization and outcomes. CA Cancer J Clin 2008; 58(1):9-31.

15. Brito C, Portela MC, Vasconcellos MTL. Sobrevida de mulheres tratadas por câncer de mama no estado do Rio de Janeiro. Rev Saude Publica 2009; 43(3):481-489.

16. Woods LM, Rachet B, Coleman MP. Origins of socioeconomic inequalities in cancer survival: a review. Ann Oncol 2006; 17(1):5-19.

17. Schneider IJC, D’orsi E. Sobrevida em cinco anos e fatores prognósticos em mulheres com câncer de mama em Santa Catarina, Brasil. Cad Saude Publica 2009; 25(6):1285-1296.

18. American Cancer Society. Cancer Treatment and Survivorship Facts \& Figures 2014-2015. Atlanta: American Cancer Society; 2014. [acessado 2016 Jun 15]. Disponível em: http://www.cancer.org/acs/groups/content/@ research/documents/document/acspc-042801.pdf.

19. Allemani C, Weir HK, Carreira H, Harewood R, Spika D, Wang XS, Bannon F, Ahn JV, Johnson CJ, Bonaventure A, Marcos-Gragera R, Stiller C, Azevedo e Silva G, Chen WQ, Ogunbiyi OJ, Rachet B, Soeberg MJ, You H, Matsuda T, Bielska-Lasota M, Storm H, Tucker TC, Coleman MP; CONCORD Working Group. Global surveillance of cancer survival 1995-2009: analysis of individual data for 25676887 patients from 279 population-based registries in 67 countries (CONCORD-2). Lancet 2015; 385(9972):977-1010.

20. Moraes AB, Zanini RR, Turchiello MS, Riboldi J, Medeiros LR. Estudo da sobrevida de pacientes com câncer de mama atendidas no hospital da Universidade Federal de Santa Maria, Rio Grande do Sul, Brasil. Cad Saude Publica 2006; 22(10):2219-2228.

21. Ricci MD, Junqueira PAA. Marcadores moleculares em câncer de mama preditivos de metástases axilares. Rev Assoc Med Bras 2008; 54(3):189.

22. Pedersen L, Gunnarsdottir KA, Rasmussen BB, Moeller $\mathrm{S}$, Lanng C. The prognostic influence of multifocality in breast cancer patients. Breast 2004; 13(3):188-193.

23. Instituto Brasileiro de Geografia e Estatística (IBGE). Cidades@. [acessado 2016 Nov 16]. Disponível em: http://www.cidades.ibge.gov.br/xtras/perfil.php?lan$\mathrm{g}=\& \operatorname{codmun}=420910 \&$ search $=$ santa-catarina|joinville|infograficos:-informacoes-completas

24. Organização das Nações Unidas (ONU). Programa das Nações Unidas para o desenvolvimento. Atlas do desenvolvimento humano. Índice de desenvolvimento humano - municipal, 1991, 2000 e 2010. ONU: New York; 2013. [acessado 2016 Nov 16]. Disponível em: http://atlasbrasil.org.br/2013/pt/ranking

25. Union for International Cancer Control (UICC). TNM Classification of malignant tumours. $7^{\text {th }}$ ed. New York: John Wiley\& Sons INC; 2010.

26. Balabram D, Turra CM, Gobbi H. Survival of patients with operable breast cancer (Stages I-III) at a Brazilian public hospital - a closer look into cause-specific mortality. BMC Cancer 2013; 13:434-443.

27. Abreu E, Koifman RJ, Fanqueiro AG, Land MGP, Koifman S. Sobrevida de dez anos de câncer de mama feminino em coorte populacional em Goiânia (GO), Brasil, 1988-1990. Cad Saúde Colet 2012; 20(3):305313. 
28. Fayer VA, Guerra MR, Cintra JRD, Bustamante-Teixeira MT. Sobrevida de dez anos e fatores prognósticos para o câncer de mama na região Sudeste do Brasil. Rev Bras Epidemiol 2016; 19(4):766-778.

29. Boada LBGL. Factores pronósticos anatomopatológicos de supervivência en el cáncer de mama. MEDISAN 2012; 16(12):1899-1905.

30. Ayala ALM. Sobrevida de mulheres com câncer de mama, de uma cidade no sul do Brasil. Rev Bras Enferm 2012; 65(4):566-570.

31. Migowski A. A interpretação das novas diretrizes para a detecção precoce do câncer de mama no Brasil. Cad Saude Publica 2016; 32(8):1-5.

32. Bleyer A, Welch G. Effect of Three Decades of Screening Mammography on Breast-Cancer Incidence. $N$ Engl J Med 2012; 367(21):1998-2005.

33. Flores-Luna L, Salazar-Martínez E, Duarte-Torres RM, Torres-Mejía G, Alonso-Ruiz P, Lazcano-Ponce E. Factores pronósticos relacionados com La supervivência Del cáncer de mama. Salud Pública Mex 2008; 50(2):119-125.

34. Fremgen AM, Bland KL, McGinnis LS, Eyre HJ, McDonald CJ, Menck HR, Murphy GP. Clinical highlights from the national cancer database. CA Cancer J Clin 1999; 49(3):145-158.

35. Batschauer APB. Avaliação hemostática e molecular em mulheres com câncer de mama receptor hormonal negative [tese]. Belo Horizonte: Universidade Federal de Minas Gerais; 2009.

36. Yoshida M, Shimizu C, Fukutomj T, Tsuda H, Kinoshita T, Akashi-Tanaka S, Ando M, Hojo T, Fujiwara Y. Prognostic factors in young japanese women with breast cancer: prognostic value of age at diagnosis. Jpn J Clin Oncol 2011; 41(2):180-189.

37. Abreu E, Koifman S. Fatores prognósticos no câncer da mama feminina. Rev Bras Cancerol 2002; 48(1):113131.

38. Eisenberg ALM. Sobrevida de cinco anos para pacientes com carcinoma ductalinfiltrante de mama sem comprometimento de linfonodos axilares. Coorte hospitalar, 1992-1996, Rio de Janeiro, Brasil [tese]. Rio de Janeiro: Fiocruz; 2004.

39. Mohammed RA, Ellis IO, Mahmmod AM, Hawkes CE, Green AR, Rakha EA, Martin SG. Lymphatic and blood vessels in basal and triple-negative breast cancers: characteristics and prognostic significance. Mod Pathol 2011; 24(6):774-785.

40. Marinho VFZ, Konradin M, Sanches FSF, Rocha GFS, Gobbi H. Marcadores moleculares em câncer de mama preditivos de metástases axilares. Rev Assoc Med Bras 2008; 54(3):203-207.

41. Hwang KT, Kim YA, Kim J, Chu AJ, Chang JH, Oh SW, Hwang KR, Chai YJ. The influences of peritumoral lymphatic invasion and vascular invasion on the survival and recurrence according to the molecular subtypes of breast cancer. Breast Cancer Res Treat 2017; 163(1):71-82.

42. Lauria R, Perrone F, Carlomagna C, De Laurentis M, Morabit A, Gallo C, Varriale E, Pettinato G, Panico L, Petrella G, et al. The prognostic value of lymphatic and blood vessel invasion in operable breast cancer. Cancer 1995; 76(10):1772-1778.

43. Kato T, Kameoka S, Kimura T, Nishikawa T, Kobayashi M. Blood vessel invasion as a predictor of long-term survival for Japanese patients with breast cancer. Breast Cancer Res Treat 2002; 73(1):1-12.
44. Dutra MC, Rezende MA, de Andrade VP, Soares FA, Ribeiro MV, Paula EC, Gobbi H. Imunofenótipo e evolução do câncer de mama: entre mulheres muito jovens e mulheres na pós-menopausa. Rev Bras Ginecol Obstet 2009; 31(2):54-60.

45. Anel A, Reed M, Leonard R, Kunkler I, Muss H, Wildiers H, Fallowfield L, Jones A, Coleman R. The treatment of early breast cancer in women over the age of 70. Br J Cancer 2011; 105(2):189-193.

46. Schonberg MA, Marcantonio ER, Li D, Silliman RA, Ngo L, McCarthy EP. Breast Cancer Among the Oldest Old: Tumor Characteristics, Treatment Choices, and Survival. J Clin Oncol 2010; 28(12):2038-2045.

47. Nixon AJ, Neuberg D, Hayes DF, Gelman R, Connolly JL, Schnitt S, Abner A, Recht A, Vicini F, Harris JR. Relationship of patient age to pathologic features of the tumor and prognosis for patients with stage I or II breast cancer. J Clin Oncol 1994; 12(5):888-894.

48. Maggard MA, O'Connell JB, Lane KE, Liu JH, Etzioni DA, Ko CY. Do young breast cancer patients have worse outcomes? J Surg Res 2003; 113(1):109-113.

49. Kataoka A, Iwamoto T, Tokunaga E, Tomotaki A, Kumamaru H, Miyata H, Niikura N, Kawai M, Anan K, Hayashi N, Masuda S, Tsugawa K, Aogi K, Ishida T, Masuoka $\mathrm{H}$, Iijima K, Kinoshita T, Nakamura S, Tokuda Y. Young adult breast cancer patients have a poor prognosis independent of prognostic clinicopathological factors: a study from the Japanese Breast Cancer Registry. Breast Cancer Res Treat 2016; 160(1):163-172.

50. Barchielli A, Balzi D. Age at diagnosis, extent of disease and breast cancer survival: a population-based study in Florence, Italy, Itália. Tumori 2000; 86(2):119-123.

51. Silva PFS, Amorim MHC, Zandonade E, Gomes KC. Associação entre variáveis sociodemográficas e estadiamento clínico avançado das neoplasias da mama em hospital de referência no Estado do Espírito Santo. Rev Bras Cancerol 2013; 59(3):361-367.

52. Pinto IV, Ramos DN, Costa MCE, Ferreira CBT, Rebelo MS. Completude e consistência dos dados dos registros hospitalares de câncer no Brasil. Cad Saúde Colet 2012; 20(1):113-20.

53. Santos SBL, Koch HA. Análise do Sistema de Informação do Programa de Controle do Câncer de Mama (SISMAMA) mediante avaliação de 1.000 exames nas cidades de Barra Mansa e Volta Redonda. Radiol Bras 2010; 43(5):295-301.

54. Estado de Santa Catarina. Secretaria de Estado da Saúde (SESSC). Indicadores de Dados Básicos RIPSA/SC. Florianópolis: SESSC; 2014.

Artigo apresentado em 13/11/2016

Aprovado em 23/07/2017

Versão final apresentada em 25/07/2017 